\title{
Mapas Conceituais de Projeto: uma ferramenta para projetar objetos de aprendizagem significativa
}

\author{
Project Conceptual Maps: a tool \\ for designing meaningful learning objects
}

\author{
Alberto Bastos do Canto Filho ${ }^{1}$. José Valdeni de Lima² . \\ Liane Margarida Rockenbach Tarouco ${ }^{3}$
}

\begin{abstract}
Resumo: Este artigo apresenta uma metodologia de projeto de objetos de aprendizagem significativa chamada Projeto Baseado em Trajetórias de Aprendizagem (PBTA). Esta metodologia considera uma metáfora onde os conhecimentos prévios do estudante são pontos de partida, os objetivos educacionais são pontos de chegada e objetos de aprendizagem são estradas que conectam os pontos de partida aos pontos de chegada. Um tipo diferenciado de Mapa Conceitual chamado Mapa Conceitual de Projeto é utilizado como ferramenta de projeto para definir relações entre o conhecimento prévio do estudante e os objetivos educacionais. A base teórica inclui a Teoria da Aprendizagem Significativa e a Teoria da Carga Cognitiva. Estudos anteriormente realizados são citados como evidências da eficácia da metodologia proposta.
\end{abstract}

Palavras-chave: Objeto de aprendizagem. Aprendizagem significativa. Mapa conceitual.

\begin{abstract}
This paper presents a methodology designed for a project on meaningful learning objects called Project Based on Learning Trajectories (PBLT). This methodology considers a metaphor where the student's previous knowledge are starting points, the educational objectives are considered destination points and the learning objects are roads connecting the starting points to destination points. A differentiated Conceptual Map called Project's Conceptual Map is a tool intended to define relations between the previous knowledge of the students (starting points) and the educational objectives (destination points). The theoretical basis of this proposed method includes Meaningful Learning Theory and Cognitive Load Theory. Previous studies are referenced as evidence of the proposed methodology's effectiveness.
\end{abstract}

Keywords: Learning object. Meaningful learning. Conceptual map.

\footnotetext{
${ }^{1}$ Universidade Federal do Rio Grande do Sul (UFRGS), Escola de Engenharia, Departamento de Engenharia Elétrica, Porto Alegre, RS, Brasil. E-mail: <alberto.b.c.f@gmail.com>.

${ }^{2}$ UFRGS, Centro Interdisciplinar de Novas Tecnologias na Educação, Porto Alegre, RS, Brasil.

${ }^{3}$ UFRGS, Faculdade de Educação, Porto Alegre, RS, Brasil.
} 


\title{
Introdução
}

Nos últimos anos o Brasil vem tendo um expressivo aumento da oferta de vagas no ensino superior. O programa REUNI, de expansão e reestruturação das universidades federais, resultou num incremento de 14.826 vagas no ensino superior, entre 2007 e 2008 (MEC / SESU / DIFES, 2009). Nos cursos de engenharia, este aumento de vagas propiciou o ingresso de um grupo de estudantes que não se classificaria na condição anterior, implicando o ingresso de um maior percentual de estudantes com lacunas formativas, especialmente nas disciplinas iniciais de matemática e física (LODER, 2009). Como consequência, houve um aumento dos índices de retenção e evasão e o número de engenheiros formados não está crescendo na mesma proporção do aumento do número de vagas (CANTO, 2011).

O processo de ensino tradicionalmente utilizado nos cursos de engenharia (aula expositiva) tem-se consolidado em função da existência de um equilíbrio adequado entre resultados alcançados e recursos demandados. Este processo de ensino está associado à aprendizagem por recepção, que é bastante eficiente quando se consideram estudantes que tenham alcançado os estágios mais avançados de maturidade cognitiva (AUSUBEL; NOVAK; HANESIAN, 1978). Infelizmente, existe uma parcela relevante de estudantes que, não obstante tenham ingressado num curso superior, ainda não alcançaram o nível de maturidade cognitiva adequado para o aproveitamento deste processo de ensino e aprendizagem.

Por outro lado, o processo de aprendizagem por descoberta possibilita ganhos de eficácia educacional por atingir também os estudantes com menor maturidade cognitiva. No entanto, este processo demanda mais tempo. Prince e Felder (2006) citam que a demanda de tempo adicional é um dos principais obstáculos ao uso mais intenso do processo de aprendizagem por descoberta:

\begin{abstract}
$\mathrm{Na}$ forma mais pura deste método, o professor especifica problemas e fornece realimentação sobre o esforço do estudante, mas não direciona ou guia estes esforços. Existem muitas razões pelas quais raramente se utiliza este método na educação superior, entre elas, o fato dos professores temerem - provavelmente com razão - que eles somente possam cobrir uma pequena parte do conteúdo previsto, caso os estudantes tenham que descobrir tudo sozinhos. (PRINCE; FELDER, 2006, p. 132, tradução nossa).
\end{abstract}

Benjamin Bloom (1984) realizou um estudo constatando que a tutoria individual é capaz de obter resultados médios dois desvios padrões (dois sigmas) acima dos resultados médios alcançados através do sistema convencional (aula expositiva em turmas com 30 estudantes). Esta grande diferença de resultados está associada aos diferentes níveis de personalização e interação. Infelizmente, não obstante a sua comprovada eficácia, a tutoria individual é uma solução que não pode ser adotada em larga escala devido aos seus elevados custos. É importante salientar aqui que uma comunicação personalizada entre professor e cada aluno é inviável pela quantidade de tempo necessária (KIRSCH-PINHEIRO; DE LIMA; BORGES, 2003). Logo, somente com o uso de Tecnologia de Informação é possível esta personalização dando mais autonomia para o aluno. 
Os Mapas Conceituais de Projeto aqui apresentados foram desenvolvidos num contexto de pesquisa sobre a possibilidade de incorporar algumas práticas da tutoria individual e da aprendizagem por descoberta em larga escala e de forma economicamente sustentável. Durante o seu desenvolvimento foram realizadas avaliações nas quais se constatou que objetos de aprendizagem desenvolvidos com este método são capazes de obter resultados melhores que aqueles obtidos em aulas expositivas tradicionais (CANTO; DE LIMA; TAROUCO, 2014).

O objetivo deste artigo é a apresentação e discussão relativa ao uso de mapas conceituais de projeto no desenvolvimento da metodologia PBTA, incluindo exemplos de como usá-los no design de sequências didáticas. $\mathrm{O}$ artigo está estruturado nas seguintes seções:

- Fundamentos Epistemológicos: fundamentam a metodologia proposta através das teorias utilizadas;

- Metodologia de Projeto: apresenta a metodologia de Projeto Baseado em Trajetórias de Aprendizagem (PBTA);

- Avaliação da Metodologia: apresenta uma referência a experimentos realizados para validação da metodologia;

- Resultados e Conclusões: apresenta os resultados dos experimentos, conclusões e trabalhos futuros.

\section{Fundamentos Epistemológicos}

Ausubel, Novak e Hanesian (1978, p. 34) definem Aprendizagem Significativa como “[...] o processo através do qual uma nova informação (um novo conhecimento) se relaciona de maneira não arbitrária e substantiva à estrutura cognitiva do estudante".

A metáfora de que a estrutura cognitiva prévia do estudante seja o ponto de partida e que os conceitos e proposições relacionados aos objetivos educacionais sejam o ponto de chegada traz consigo a ideia de uma trajetória de aprendizagem significativa. Neste contexto, um Objeto de Aprendizagem é um caminho, que pode ser utilizado por qualquer estudante que esteja no ponto de partida e deseje chegar ao ponto de chegada (CANTO et al., 2014). A metodologia de Projeto Baseado em Trajetórias de Aprendizagem (PBTA) utiliza esta metáfora, definindo como uma de suas premissas fundamentais de projeto o Princípio dos Subsunçores.

O objetivo primordial de qualquer Objeto de

Aprendizagem (OA) é a aprendizagem significativa, isto é, o estabelecimento de relações não arbitrárias e substantivas entre os novos conhecimentos e a estrutura cognitiva do estudante.

Um dos fatores responsáveis pela eficácia da tutoria individual é a possibilidade de uma abordagem personalizada em função da estrutura cognitiva individual. Este nível de personalização é muito difícil em aulas expositivas onde as proposições são apresentadas para um grande número de estudantes que não possuem a mesma estrutura cognitiva. $\mathrm{O}$ uso de tecnologias que facilitem a aplicação de testes de conhecimento permite a identificação das diferentes estruturas 
cognitivas dos alunos e a definição de trajetórias individuais, implementando em larga escala este tipo de personalização. A Figura 1 mostra como isto é feito na metodologia PBTA: (a) em tempo de projeto definem-se os conceitos subsunçores nos quais serão ancoradas as novas proposições apresentadas pelo objeto de aprendizagem. Definem-se também testes que avaliam se o estudante realmente conhece estes conceitos; e (b) em tempo de execução, o estudante executa os testes e, caso seja diagnosticada a falta de um conceito subsunçor essencial, o estudante passa a executar outro OA, cujo objetivo educacional é a aprendizagem deste conceito faltante.

Figura 1. Especificação e execução de OAs

a. Tempo de Projeto

b. Tempo de Execução
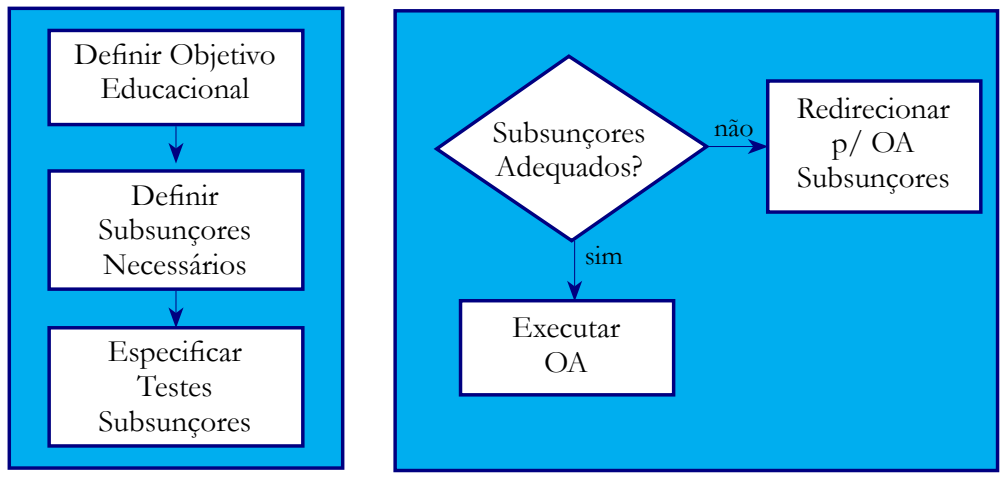

Fonte: Canto et al. (2014).

Objetos de Aprendizagem são projetados e utilizados com um objetivo: o objetivo educacional. No domínio cognitivo, os objetivos educacionais podem ser estabelecidos considerando diferentes níveis de complexidade e graus de abstração, indo desde a memorização factual até capacitação para a criação, conforme propõe a Taxonomia de Bloom (BLOOM; KRATHWOHL, 1956; KRATHWOHL, 2002). No escopo investigado neste trabalho (cursos de engenharia) se trabalha com objetivos educacionais de capacitação para o projeto, o nível de maior complexidade na taxonomia de Bloom, que exige a aprendizagem profunda dos conceitos abordados. Cabe ressaltar que a diferenciação entre a Aprendizagem Memorística e a Aprendizagem Significativa não exclui a relevância da memorização e retenção do conhecimento, pois, como não é possível separar um conceito de sua representação simbólica (LABURÚ; BARROS; SILVA, 2011), a memorização de símbolos e convenções utilizadas na engenharia sempre estará implicitamente presente nos objetivos educacionais de aprendizagem significativa.

A Teoria da Carga Cognitiva (SWELLER, 1988) apresenta aspectos relacionados à arquitetura cognitiva humana e suas limitações. Segundo esta teoria, os processamentos conscientes ocorrem na memória de trabalho, que é limitada quanto ao número de elementos de informação tratados simultaneamente. Este limite situa-se num patamar entre sete e nove elementos simultâneos, mas se reduz para dois a quatro elementos simultâneos quando há necessidade de 
Mapas Conceituais de Projeto: uma ferramenta para projetar objetos ...

processamentos típicos da aprendizagem significativa tais como organizar, comparar, etc. (VAN MERRIËNBOER; SWELLER, 2010).

Ao considerar que os Objetos de Aprendizagem Significativa exigem intenso processamento cognitivo, deve-se avaliar a condição mais restritiva da memória de trabalho (dois a quatro elementos de informação simultâneos). Isto é, na condição extrema o projeto deve considerar apenas dois elementos de informação simultâneos: um conceito subsunçor e uma nova proposição, apresentada pelo OA. Caso uma nova proposição seja apresentada antes do estudante relacionar nova proposição e o conceito subsunçor ocorrerá uma sobrecarga cognitiva e, consequentemente, perda no processo de aprendizagem. Em outras palavras, o projeto de Objetos de Aprendizagem deve levar em consideração a variável tempo, garantindo que a apresentação de cada proposição somente ocorra após o processamento da proposição precedente. Esta necessária administração do fluxo de proposições é apresentada como o "Princípio do Ajuste de Ritmo". (CANTO et al., 2013).

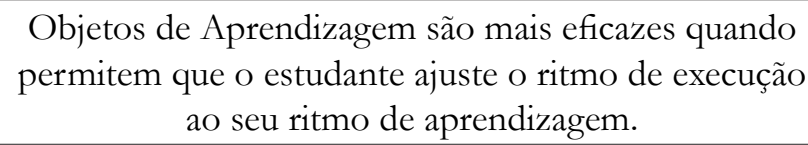

A metodologia PBTA utiliza diversos outros princípios de projeto fundamentados na Teoria da Carga Cognitiva (CANTO; DE LIMA; TAROUCO, 2014; MAYER, 2008; MAYER; MORENO, 2003; MORENO; MAYER, 2007), que não serão aqui abordados por estarem fora do escopo central deste artigo.

Na seção a seguir, serão apresentados os Mapas Conceituais de Projeto utilizados pela metodologia PBTA na etapa de projeto.

\section{Metodologia de Projeto Baseado em Trajetórias de Aprendizagem (PBTA)}

A metodologia PBTA tem por objetivo o Projeto de Objetos de Aprendizagem Significativa, e será apresentada nesta seção como a seguinte sequência de etapas: (1) Criação do Mapa Conceitual de Projeto; (2) Especificação dos Pontos de Chegada; (3) Especificação dos Pontos de Partida; (4) Definição dos Pontos de Passagem; (5) Segmentação Interobjetos; (6) Segmentação em Proposições Base; (7) Definição da Ordem de Apresentação; (8) Especificação das Proposições de Projeto; e (9) Implementação do Objeto de Aprendizagem.

Cada uma destas etapas é apresentada numa subseção a seguir, utilizando um exemplo ilustrativo em que se deseja projetar um objeto de aprendizagem, cujo objetivo seja o entendimento do conceito de velocidade variável e derivada. Na seção a seguir será apresentada a primeira etapa: criação do Mapa Conceitual de Projeto.

\section{Criação do Mapa Conceitual de Projeto}

Tavares (2007, p. 72) define Mapa Conceitual como "uma estrutura esquemática para representar um conjunto de conceitos imersos numa rede de proposições”. Segundo o autor, "[...] existe uma grande variedade de tipos de mapas disponíveis [...]. No entanto, o único tipo de mapa que explicitamente utiliza uma teoria cognitiva em sua elaboração é o mapa hierárquico do tipo proposto por Novak e Gowin (1998, 1999)" (TAVARES, 2007, p. 75). 
Na metodologia PBTA propõe-se a utilização de uma modalidade diferenciada de mapa conceitual, aqui designada Mapa Conceitual de Projeto (MCP). Este instrumento é um tipo particular de Mapa Conceitual que, não obstante esteja baseado na Aprendizagem Significativa, caracteriza-se por admitir não apenas relações hierárquicas baseadas em diferenciações progressivas ou reconciliações integrativas, mas também outros tipos de relações entre os conceitos presumidamente conhecidos e os conceitos e proposições relacionados aos objetivos educacionais. Por este motivo, ferramentas utilizadas para a construção de Mapas Conceituais (por exemplo, CmapTools) podem ser utilizadas como ferramentas de apoio ao projeto.

Como exemplo ilustrativo, o projeto de um OA cujo objetivo seja "entendimento do conceito de Velocidade Variável" é iniciado criando-se um Mapa Conceitual de Projeto com a seguinte questão focal: "Que conceitos e proposições conhecidos pelo estudante se relacionam com os conceitos de velocidade variável e derivada?".

A etapa seguinte à criação do Mapa Conceitual de Projeto é a especificação dos pontos de chegada, que será apresentada na próxima subseção.

\section{Especificação dos Pontos de Chegada}

A Figura 2 apresenta uma tela do sistema ABCAutoria, desenvolvido no projeto de pesquisa que criou a metodologia PBTA. A Figura 2 mostra a mecânica de especificação de objetivos educacionais (pontos de chegada), com base na Taxonomia Modificada de Bloom (KRATHWOHL, 2002). Os detalhes constantes na descrição do objetivo educacional permitem identificar com maior clareza os conceitos e proposições que devem ser adicionados ao Mapa Conceitual de Projeto como pontos de chegada.

Figura 2. Cadastro de Ponto de Chegada

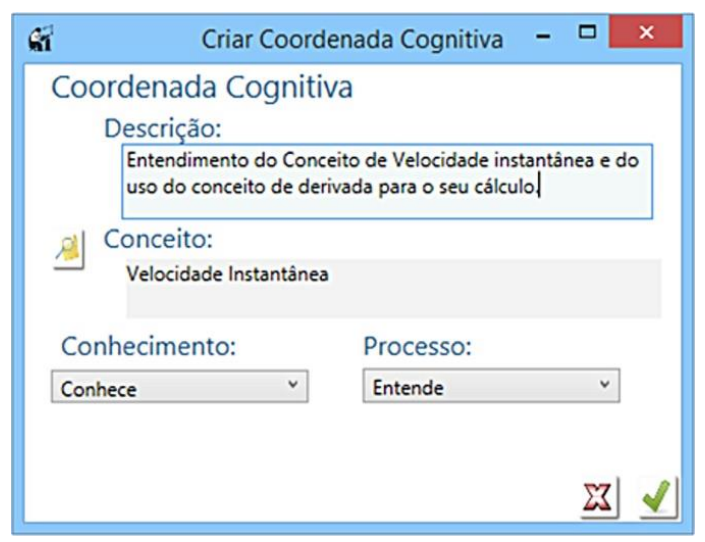

Fonte: elaborada pelos autores.

A Taxonomia de Bloom (BLOOM; KRATHWOHL, 1956) foi concebida como um meio de facilitar a troca de itens de testes entre faculdades de várias universidades, de forma a criar um banco de itens destinados à mensuração de objetivos comuns. Na metodologia PBTA 
o estabelecimento de Objetivos Educacionais e o desenvolvimento de um sistema de avaliação são feitos simultânea e interativamente. Para exemplificar este tipo de interação, considere que a questão apresentada na Figura 3 seja utilizada para avaliar a proximidade do ponto de chegada especificado na Figura 2.

Figura 3. Avaliação - Velocidade Variável

$$
\begin{aligned}
& \text { Considerando que a equação abaixo modela a } \\
& \text { distância percorrida em função do tempo, qual a } \\
& \text { velocidade no instante } t=3 \text { ? } \\
& \qquad d(t)=7 t^{2}-3 t+50
\end{aligned}
$$

Fonte: elaborada pelos autores.

Observe que há uma inconsistência entre o objetivo educacional e o sistema de avaliação: a questão apresentada exige um nível de conhecimento mais complexo do que simplesmente conhecer e entender velocidade variável e derivadas: esta questão poderia ser utilizada para avaliar se o estudante aplica métodos de cálculo diferencial ao conceito de velocidade variável. A especificação simultânea do objetivo educacional e do sistema de avaliação permite identificar e corrigir este tipo de inconsistência.

Após a especificação dos pontos de chegada e sistema de avaliação correspondente, são especificados os Pontos de Partida, conforme apresenta a subseção a seguir.

\section{Especificação dos Pontos de Partida}

Pontos de partida são conceitos e proposições presumidamente conhecidos pelo estudante, que servirão de base a partir da qual o Objeto de Aprendizagem estabelecerá relacionamentos com os objetivos educacionais. A mecânica de especificação dos Pontos de partida é similar à especificação de objetivos educacionais (Pontos de chegada) apresentados seção anterior, exceto pelo fato de que os pontos de partida são objetivos educacionais presumidamente já alcançados pelos estudantes.

O sistema de avaliação dos conceitos e proposições presumidamente conhecidos (proximidade do ponto de partida) tem o objetivo de definir o público alvo do OA, constituído por estudantes que efetivamente conheçam os conceitos e proposições presumidamente conhecidas. Através desta avaliação, é possível definir trajetórias de aprendizagem individuais, conforme mostra a Figura 1.

Nesta etapa, deve-se considerar que "a aprendizagem científica e o posicionamento crítico dos alunos em relação às estratégias locais de identidade são favorecidos pela ancoragem sociocultural dos conteúdos" (ALMEIDA, 2012, p. 189); em contrapartida, a ancoragem regionalizada tenderá reduzir a o público alvo, pois o OA não terá significado lógico para estudantes não pertencentes à realidade sociocultural considerada.

Após a especificação de Pontos de Chegada e Pontos de Partida, o Mapa Conceitual de Projeto deve ser completado com o acréscimo de Pontos de Passagem, conforme apresenta a subseção a seguir. 


\section{Definição dos Pontos de Passagem}

Após a execução das etapas descritas nas seções acima, o Mapa Conceitual de Projeto possuirá dois conjunto de conceitos e proposições: pontos de partida e pontos de chegada. Nesta etapa, devem-se adicionar proposições que estabeleçam relacionamentos entre os conceitos pertencentes ao conjunto de Pontos de Partida e os conceitos pertencentes ao conjunto de pontos de chegada, respondendo à pergunta: como os conceitos e proposições presumidamente conhecidos se relacionam com os conceitos e proposições relacionados aos objetivos educacionais?

Eventualmente, pode não ser possível estabelecer uma relação direta entre os pontos de partida e os pontos de chegada, havendo a necessidade de estabelecer objetivos educacionais parciais aqui denominados de pontos de passagem.

A Figura 4 mostra um exemplo de Mapa Conceitual de Projeto cuja questão focal seja identificar conceitos e proposições presumidamente conbecidos pelo estudante que se relacionem com os conceitos de velocidade variável e derivada. Observe a existência de diversos pontos de passagem, o que implicaria a construção de um Objeto de Aprendizagem que abordasse um grande número de novos conceitos e proposições. Esta situação nem sempre é conveniente, pois resulta em projetos complexos e OAs extensos, com poucas possibilidades de reutilização.

Figura 4. Mapa Conceitual de Projeto: velocidade variável

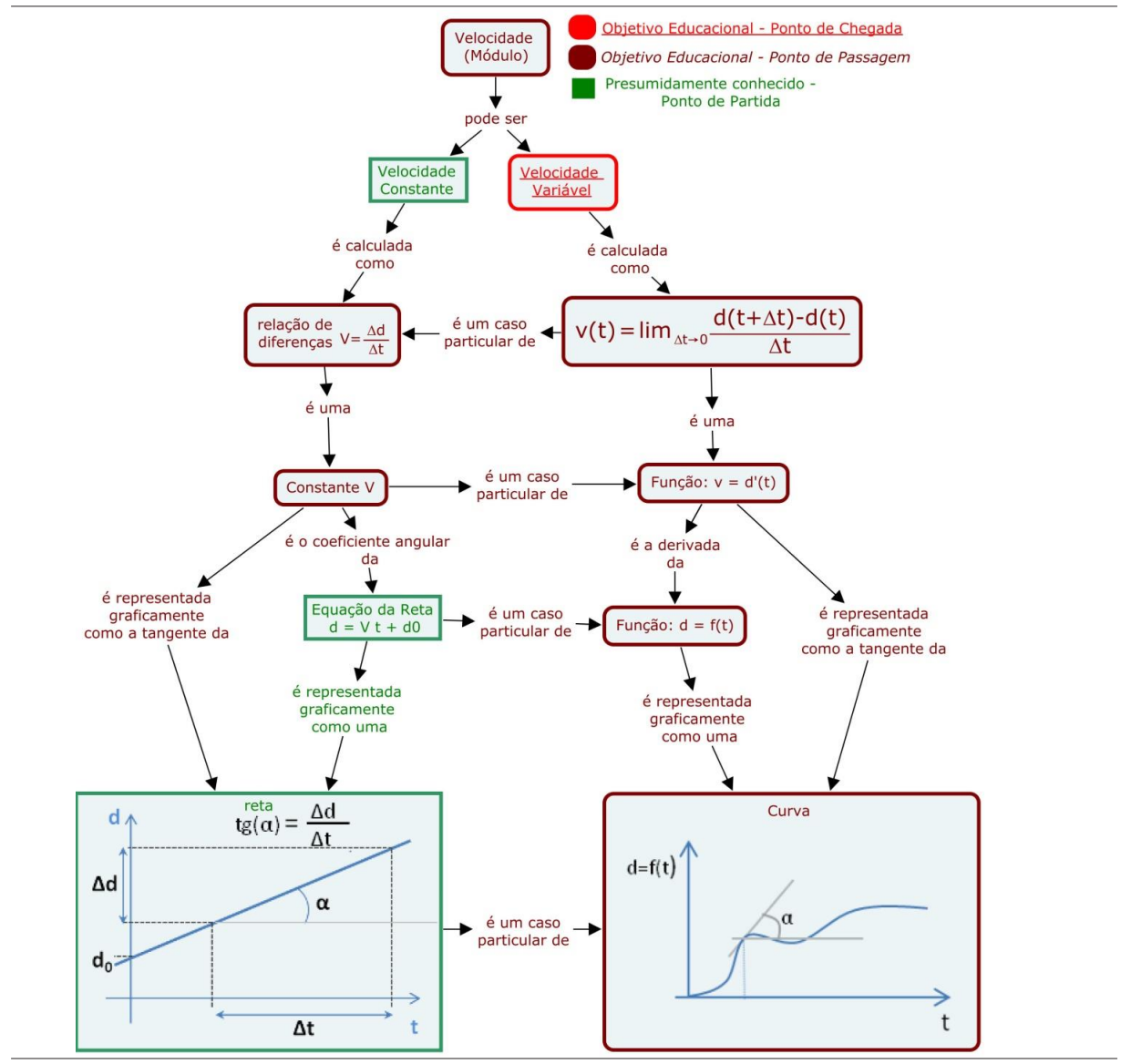

Fonte: elaborada pelos autores. 
A Segmentação Interobjetos apresentada na próxima subseção é a estratégia adotada para resolver esta situação.

\section{Segmentação Interobjetos}

Segmentação Interobjetos é a subdivisão do projeto de um Objeto de Aprendizagem em diversos subprojetos. Cada um destes subprojetos é tratado como um projeto independente, que possui objetivos educacionais próprios, definidos a partir do projeto original. Por exemplo, a Figura 5, a seguir, mostra um exemplo de Mapa Conceitual de Projeto criado para abordar o conceito de relação de diferenças. Este mapa refere-se ao projeto de um novo OA, e foi construído segundo os passos descritos nas subseções anteriores, considerando o objetivo educacional de capacitar o estudante para resolução de problemas que envolvam o conceito de relação de diferenças.

Figura 5. Mapa Conceitual de Projeto: relações de diferenças

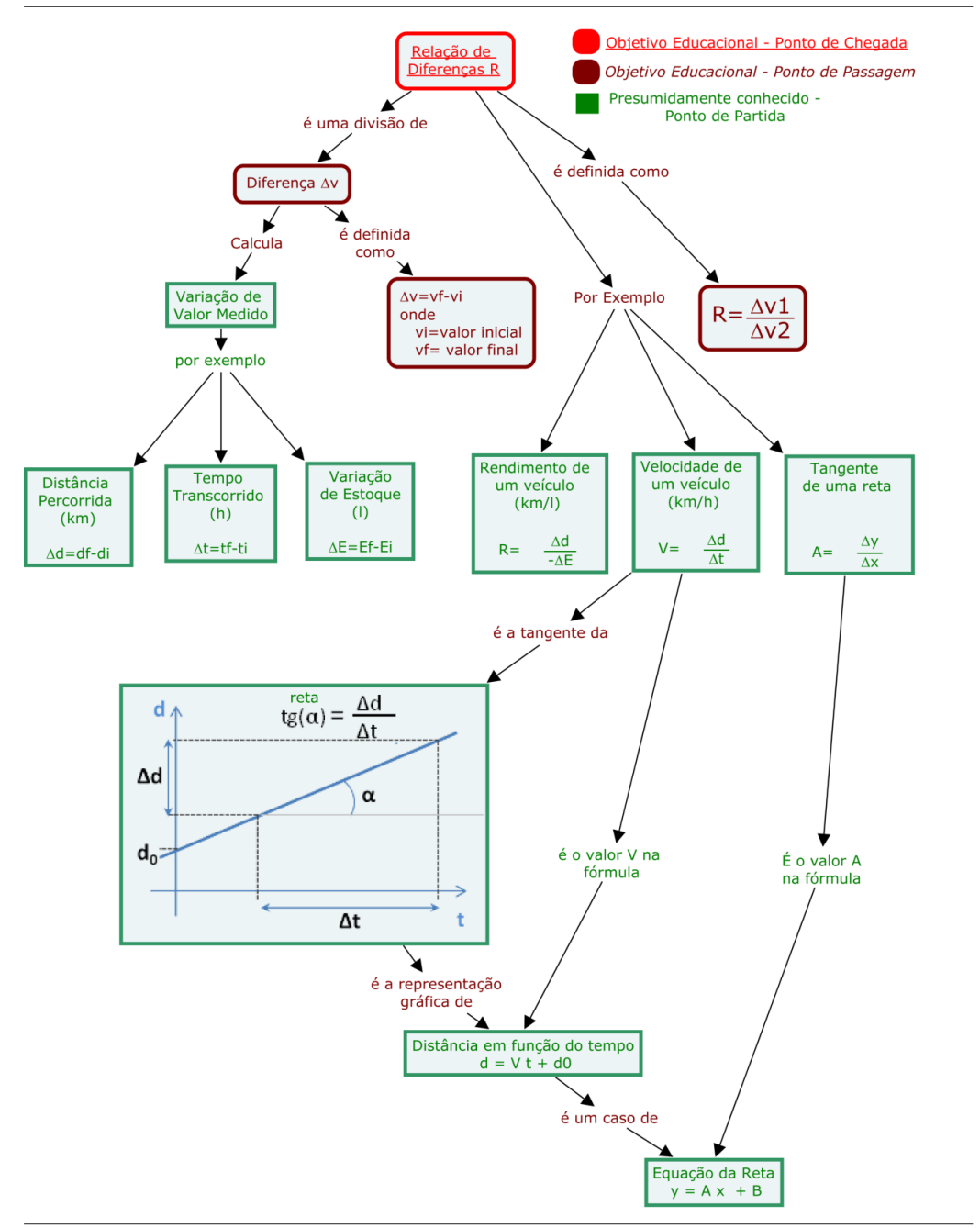

Fonte: elaborada pelos autores. 
Observe na Figura 5 que o Mapa Conceitual de Projeto apresenta diversos pontos de partida que não constavam no mapa original, mostrando um maior detalhamento sobre os subsunçores nos quais as novas proposições serão ancoradas. Observe também a premissa que o estudante conhece os conceitos de velocidade constante e reta, mas que desconhece a relação existente entre eles.

Assumindo-se que o OA sobre relaçôes de diferenças (Figura 5) seja executado anteriormente ao OA sobre velocidade variável, deve-se modificar o Mapa Conceitual de Projeto mostrado na Figura 4, pois diversos conceitos e proposições passarão a ser identificados como "presumidamente conhecidos". O Mapa Conceitual de Projeto apresentado na Figura 5 será utilizado como exemplo na próxima subseção, que descreve a forma como as proposições base são extraídas do Mapa Conceitual de Projeto.

\section{Segmentação em Proposições Base}

O objetivo desta etapa de projeto é extrair Sequências de Proposições Base (SPB) do mapa conceitual de projeto. O texto das proposições base deve iniciar com um conceito previamente conhecido ou previamente abordado numa Proposição Base precedente. Esta mecânica é exemplificada nas SPBs mostradas no Quadro 1 e no Quadro 2 (listas extraídas do Mapa Conceitual de Projeto mostrado na Figura 5).

Quadro 1. SPB1: velocidade constante e a tangente da reta

\begin{tabular}{|c|c|c|c|}
\hline 1. & $\begin{array}{l}\text { Tangente de uma reta } \\
\qquad A=\frac{\Delta y}{\Delta x}\end{array}$ & é o valor A na & $\begin{array}{l}\text { Equação da Reta } \\
\qquad y=\mathrm{Ax}+\mathrm{B}\end{array}$ \\
\hline 2. & $\begin{array}{l}\text { Velocidade de um Veículo } \\
(\mathrm{km} / \mathrm{h}) \\
\qquad V=\frac{\Delta d}{\Delta t}\end{array}$ & é o valor $\mathrm{V}$, na fórmula & $\begin{array}{l}\text { Distância em função do } \\
\text { tempo } \\
\qquad \mathrm{d}=\mathrm{Vt}+\mathrm{d}_{0}\end{array}$ \\
\hline 3. & $\begin{array}{l}\text { Distância em função do tempo } \\
\qquad \mathrm{d}=\mathrm{Vt}+\mathrm{d}_{0}\end{array}$ & é um caso de & $\begin{array}{l}\text { Equação da Reta } \\
\qquad y=A x+B\end{array}$ \\
\hline 4. & $\begin{array}{l}\text { Distância em função do tempo } \\
\qquad \mathrm{d}=\mathrm{Vt}+\mathrm{d}_{0}\end{array}$ & $\begin{array}{l}\text { é representada graficamente como } \\
\text { uma }\end{array}$ & Reta \\
\hline 5. & $\begin{array}{l}\text { Velocidade de um Veículo } \\
(\mathrm{km} / \mathrm{h}) \\
\qquad V=\frac{\Delta d}{\Delta t}\end{array}$ & é a tangente da & $\begin{array}{l}\text { Reta } \\
\text { (gráfico distância X } \\
\text { tempo) }\end{array}$ \\
\hline
\end{tabular}

Fonte: elaborado pelos autores. 
Mapas Conceituais de Projeto: uma ferramenta para projetar objetos ...

Quadro 2. SPB2: relações de diferenças

\begin{tabular}{|c|c|c|c|}
\hline 1. & $\begin{array}{l}\text { Distância Percorrida } \\
\text { Tempo Transcorrido } \\
\text { Estoque Consumido }\end{array}$ & são exemplos de & Variação de Valor Medido \\
\hline 2. & Variação de Valor Medido & é calculado através de & Diferença $\Delta v$ \\
\hline 3. & Diferença $\Delta v$ & é definida como & $\begin{array}{l}\Delta v=v_{f}-v_{i} \\
\text { onde } \\
v_{f}: \text { valor final } \\
v_{i}: \text { valor inicial }\end{array}$ \\
\hline 4. & Diferenças & são divididas para criar & Relação de Diferenças \\
\hline 5 . & Relação de Diferenças $R$ & é definida como & $R=\frac{\Delta v_{1}}{\Delta v_{2}}$ \\
\hline 6. & $\begin{array}{l}\text { Velocidade Constante }(\mathrm{km} / \mathrm{h}) \\
\text { Rendimento de um Veículo }(\mathrm{km} / \mathrm{l}) \\
\text { Tangente de uma reta }\end{array}$ & são exemplos de & $\underline{\text { Relação de Diferenças }}$ \\
\hline
\end{tabular}

Fonte: elaborado pelos autores.

A SPB1 (Quadro 1) exemplifica a identificação de um objetivo educacional que visa a reconciliação integrativa: os conceitos de reta e de velocidade constante são presumidamente conhecidos pelo estudante, mas a integração destes dois conceitos é presumidamente desconhecida.

A SPB2, apresentada no Quadro 2, foi extraída do mapa conceitual no sentido “dos ramos para a raiz", numa abordagem de generalização que parte do conhecimento factual para o conbecimento do conceito.

As duas sequências de proposições apresentadas neste exemplo devem ser acessadas segundo uma ordem de acesso definida pelo autor do roteiro que será utilizado pelo objeto de aprendizagem, conforme apresenta a subseção a seguir.

\section{Definição da Ordem de Apresentação das Sequências de Proposições Base}

A etapa de Segmentação Intraobjeto em Proposições Base poderá gerar diversas SPB. Ao projetar um OA segundo a metodologia PBTA o projetista (responsável pelo design de sequências didáticas) deve prever a ordem de execução segundo uma das seguintes alternativas:

- Sequencial: o projetista define a ordem de execução das SPBs;

- Aleatória: o projetista define que, em tempo de execução, o estudante escolherá a ordem de acesso às SPB;

- Parcialmente Aleatório: o estudante poderá escolher a ordem de acesso a algumas SPBs; as demais possuem uma ordem predefinida pelo projetista.

A Figura 6 mostra um exemplo em que o sistema $A B C$ Autoria é utilizado para especificar uma sequência de acesso aleatório às proposições base SPB1 e SPB2, apresentadas no Quadro 1 e no Quadro 2.

A Figura 7 mostra a forma como o acesso aleatório foi implementado, permitindo que o estudante defina a sequência em tempo de execução 
Canto Filho, A. B.; Lima, J. V.; Tarouco, L. M. R.

Figura 6. Acesso Aleatório em tempo de Projeto

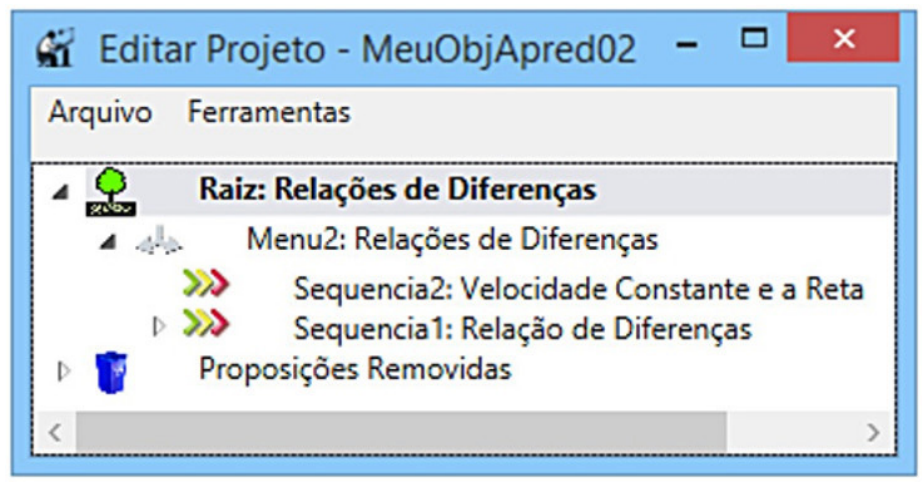

Fonte: elaborada pelos autores.

Figura 7. Acesso aleatório em tempo de execução

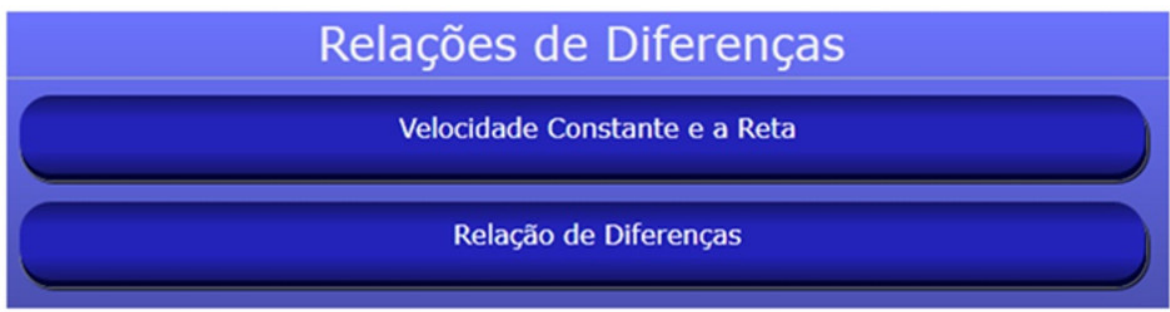

Fonte: elaborada pelos autores.

Na próxima subseção será apresentada a forma como cada proposição base é subdividida em proposições de projeto.

\section{Especificação das Proposições de Projeto}

Proposição de projeto será aqui definida como "uma proposta que o OA apresenta para o estudante com o propósito de alcançar um objetivo educacional”. Na versão atual da metodologia PBTA, são considerados os Tipos de Proposição de Projeto mostrados a seguir.

\section{Proposições Básicas:}

- Proposição Exposição: proposição que utiliza os recursos multimídia do computador (imagens estáticas, animações, vídeos, áudio e textos) para apresentar fatos, ideias, conceitos, etc.;

- Proposição Questão: Proposição exposição que apresenta uma questão que deve ser respondida pelo estudante. 


\section{Proposições de Agrupamento:}

- Proposição Sequência: Conjunto de proposições apresentadas sequencialmente. Após a apresentação de cada uma das proposições pertencentes à sequência há uma interrupção (pausa para reflexão); a proposição subsequente é apresentada quando há uma solicitação do estudante;

- Proposição Menu: Conjunto de proposições cuja execução pode ser executada sem uma ordem pré-definida; a sequência de execução das proposições é escolhida pelo estudante, em um menu de opções.

Nesta etapa do projeto, cada uma das Proposições Base é detalhada como um conjunto de proposições de projeto. As sequências do tipo resgate-questão-exposição (CANTO; DE LIMA; TAROUCO, 2014) são um caso particular de detalhamento das proposições base utilizando uma abordagem de aprendi₹agem baseada em problemas. No resgate são utilizadas proposições que trazem para a memória de trabalho os conceitos e proposições conhecidos. A questão é um problema relacionado ao objetivo educacional. Sua solução requer o entendimento de conceitos desconhecidos pelo estudante, mas que podem ser descobertos. Seu objetivo é estimular a reflexão sobre os novos conceitos. A exposição apresenta os novos conceitos e proposições.

Esta opção de aprendizagem por descoberta guiada é especialmente útil para estudantes que ainda não tenham alcançado um nível de maturidade cognitiva adequado para o aprendizado significativo através da aprendizagem por recepção pura. A Figura 8 mostra um exemplo de sequências do tipo resgate-questão-exibição, que foi extraída do Objeto de Aprendizagem "NUMBIN", desenvolvido com a metodologia PBTA visando à aprendizagem de Bases Numéricas e Números Binários.

Figura 8. Resgate-Questão-Exposição: exemplo

a. Resgate: conceito subsunçor é carregado na memória de trabalho

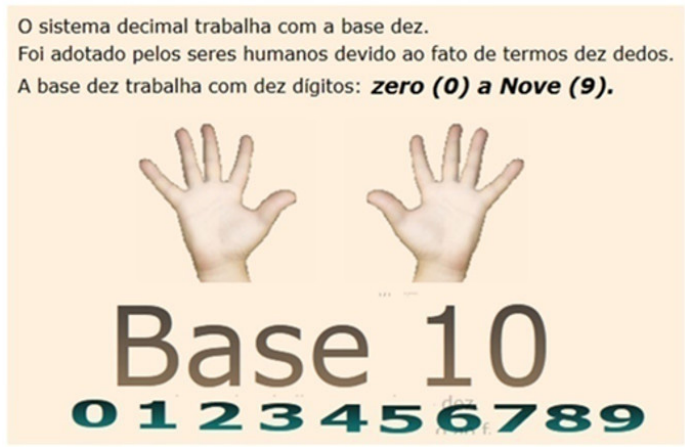

b. Questão: Proposição de uma questão instigadora, que exige um esforço cognitivo característico da aprendizagem ativa, que irá diferenciar os conceitos conhecidos

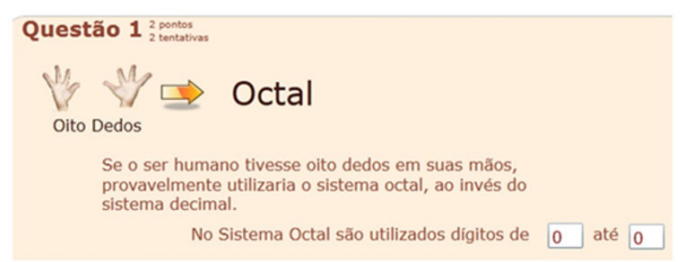

c. Exposição: apresentação de conceitos consolidados, relacionados à questão instigadora

\section{Base B Base 5}

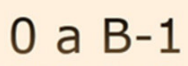

0 a 4

Num sistema numérico que utilize a Base Por exemplo, na base 5, utilizam-se $\mathrm{B}$, os dígitos utilizados vắo de 0 até $\mathrm{B}-1$. dígitos de $0 \mathrm{a} 4$.

Fonte: Elaborada pelos autores a partir do Objeto de Aprendizagem NUMBIN ${ }^{\circledR}$ 
A Figura 9 apresenta a carga cognitiva projetada ao longo do tempo, em sequências do tipo Resgate (R) - Questão (Q) - Exibição (E). O eixo vertical (carga) apresenta o número de elementos presentes na memória de trabalho; $\mathrm{O}$ eixo horizontal apresenta o tempo. Observe que a carga da memória de trabalho varia entre os valores zero (no instante inicial), 1 (memória carregada com um conceito subsunçor) e 2 (conceito subsunçor + Proposição).

Figura 9. Carga cognitiva nas sequências Resgate-Questão-Exposição

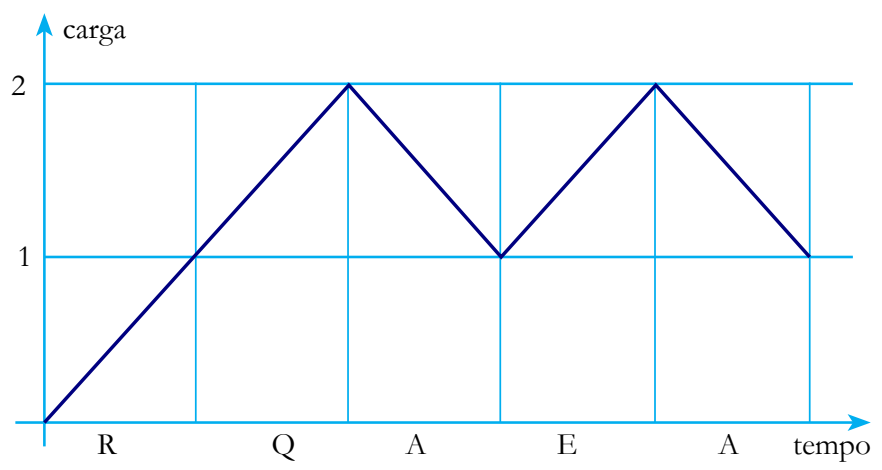

Fonte: elaborada pelos autores.

A primeira etapa nesta sequência é o Resgate (identificada pela letra R no eixo horizontal), que carrega a memória de trabalho com um elemento de informação: um conceito subsunçor. Num segundo momento é apresentada uma Questão instigadora (Letra Q, no eixo horizontal), carregando a memória de trabalho com dois elementos de informação simultâneos: o conceito subsunçor e a proposição-questão. A letra 'A' apresentada na linha de tempo representa um tempo de aprendizagem significativa, no qual o estudante realiza as operações cognitivas necessárias para o estabelecimento de relações entre os conceitos subsunçores e o novo elemento de informação. O resultado destes processamentos será a modificação dos conceitos subsunçores originais por um processo de integração ou diferenciação, após o qual resultará um único elemento de informação na memória de trabalho. Neste momento, o estudante poderá iniciar a etapa seguinte, de exposição, na qual são apresentadas proposições que expõem os conceitos relacionados à questão proposta segundo a visão consolidada pela literatura, voltando a carga para dois elementos simultâneos: a proposição exposição e os conceitos diferenciados resultantes da proposição questão. Finalmente, inicia-se uma nova etapa de aprendizagem, na qual há um novo processamento cognitivo que comparará os conceitos descobertos na resolução da questão com os conceitos consolidados recém expostos.

A especificação das Proposições de Projeto deve incluir todos os elementos necessários para a implementação (textos, áudios, imagens, vídeos, gabaritos das questões, etc.). O resultado desta etapa de projeto é um Roteiro Detalhado, que será utilizado na fase de implementação, apresentada na subseção a seguir. 


\section{Implementação do Objeto de Aprendizagem}

Nesta etapa, as especificações geradas nas etapas anteriores são utilizadas para a produção do Objeto de Aprendizagem. A tecnologia utilizada para a implementação do OA pode atuar como um elemento restritivo, eventualmente inviabilizando alguma especificação de projeto. Por exemplo, se uma determinada tecnologia for restritiva quanto à interatividade exigida pelas questões, as especificações deste tipo de proposição devem ser revistas. A etapa de implementação não será aprofundada neste artigo, cujo foco central é o uso de Mapas Conceituais de Projeto. A seção a seguir apresentará resultados e conclusões sobre a metodologia proposta.

\section{Resultados e Conclusões}

Nesta seção serão apresentadas as conclusões sobre a metodologia PBTA e os Mapas Conceituais de Projeto. Como suporte para estas conclusões são referenciados dados obtidos com o uso de um objeto de aprendizagem que foi desenvolvido segundo a metodologia proposta.

Canto, Lima e Tarouco (2014) relatam estudo realizado para avaliar a eficácia da metodologia PBTA, no qual foi desenvolvido um Objeto de Aprendizagem que aborda o tema Bases Numéricas - Números Binários. Este tema é normalmente apresentado em aula expositiva de duas horas, para estudantes dos cursos de Engenharia Elétrica e Engenharia de Controle e Automação da Universidade Federal do Rio Grande do Sul.

Foram comparados quatro grupos de dez estudantes, considerando diferentes métodos (Ensino Tradicional (ET) ou uso do Objeto de Aprendizagem (AO)) e diferentes abordagens afetivas (Aprendizagem Forçada (AF) - quando os estudantes foram informados sobre uma prova que "Valeria Nota", ou Aprendizagem Natural (AN) - quando os estudantes não foram pressionados a estudar para a prova).

Figura 10. Estudo de Caso

a. Grupos Investigados

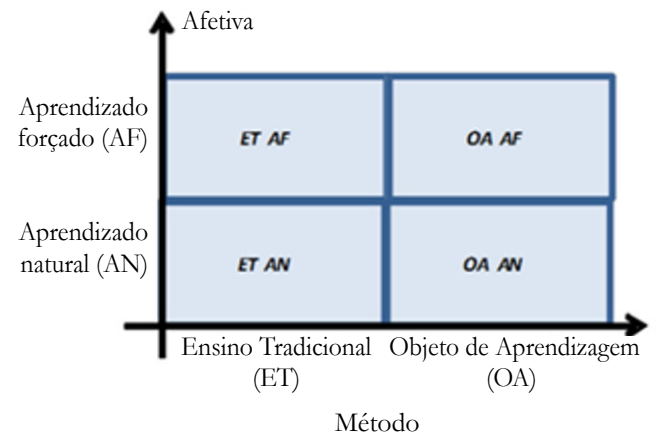

b. Média e Desvio Padrão - Teste de Conhecimento

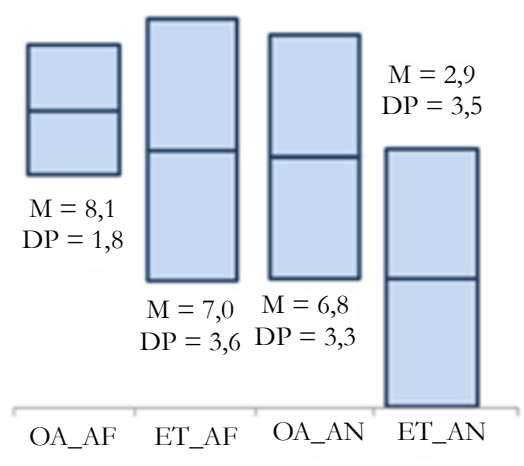

Fonte: Canto, Lima e Tarouco (2014). 
A Figura 10a mostra os quatro grupos investigados. A Figura 10b mostra as Médias e Desvios Padrões dos escores obtidos por cada um dos grupos no teste de conhecimentos.

Observe que: (a) o grupo OA_AF, que utilizou o Objeto de Aprendizagem associado a uma motivação extrínseca teve um desempenho médio mais alto (Média=8,1) e mais homogêneo (Desvio Padrão= 1,8) que os demais grupos; (b) o sistema tipicamente utilizado nos cursos de engenharia (Aulas expositivas e avaliações trimestrais - ET_AN) obteve os piores resultados; (c) o simples fato de introduzir uma avaliação como fator motivacional já ocasionou uma melhoria de desempenho (Grupo ET_AF), indicando que a qualidade do OA é apenas um dos fatores que podem influenciar o processo de aprendizagem.

Uma das vantagens do uso de objetos de aprendizagem é a facilidade para a realização de atividades interativas que estimulem a reflexão através de questões corrigidas "em tempo real". Dificilmente esta abordagem pode ser adotada em aulas tradicionais devido à demanda de horas adicionais para aplicar os testes, ao trabalho adicional para correção e aos diferentes tempos de resposta dos estudantes, que implicariam longos tempos de espera para aqueles que terminassem antes. Estudantes que possuem o hábito de "estudar para a prova" tenderiam a estudar diariamente se fossem realizadas provas diárias. O uso de tecnologia viabiliza a avaliação continuada, pois permite a realização de testes à distância com correção automática, incentivando uma mudança de hábitos de estudos que é essencial quando os conceitos abordados numa determinada aula servem de base para o entendimento dos conceitos apresentados na aula subsequente. Adicionalmente, a interatividade dos OAs é um fator que favorece a manutenção da atenção de estudantes que tenham dificuldades com os sistemas de aprendizagem por recepção pura (MORENO; MAYER, 2007).

$\mathrm{O}$ ajuste de ritmo é outro fator que favorece o uso de OAs, pois cada estudante necessita um tempo diferente para analisar e rever uma determinada proposição. Em aulas expositivas ministradas para turmas grandes o docente adota um "ritmo médio" de apresentação das proposições, que poderá ser muito acelerado para alguns estudantes e muito lento para outros, resultando em sobrecarga cognitiva ou dispersão da atenção. Além disto, a reapresentação de uma proposição numa aula expositiva é muitas vezes inviabilizada devido ao tempo limitado disponível para apresentação do conteúdo e à exposição frente à turma que ocorre sempre que um estudante solicita que um conteúdo seja reapresentado.

Aulas expositivas possuem hora e local determinados, impondo que o estudante despenda tempo de deslocamento até a sala de aula, o que não ocorre com OAs, que podem ser executados no horário e local de maior conveniência para o aprendizado.

Resumindo, Objetos de Aprendizagem possuem um conjunto possibilidades que poderão ser exploradas para a melhoria dos processos de ensino e aprendizagem, introduzindo em larga escala certo nível de personalização e uso de técnicas de aprendizagem por descoberta.

A metodologia PBTA contribui para que sejam criados OAs com potencial significativo, pois se fundamenta no estabelecimento de relações entre os objetivos educacionais e os conceitos e proposições previamente conhecidos pelo estudante, garantindo assim o principal aspecto da aprendizagem significativa: "O fator isolado mais importante que influencia a aprendizagem é aquilo que o aprendiz já conhece. Descubra o que ele sabe e baseie nisso os seus ensinamentos”. (AUSUBEL; NOVAK; HANESIAN, 1978).

Finalmente, ressalta-se que seria uma abordagem reducionista a proposição de que a simples utilização de um determinado método seja condição suficiente para a produção de OAs 
de qualidade, pois além da correta utilização do método PBTA, a qualidade do projeto depende das escolhas do projetista, que deverá especificar os objetivos educacionais, os conceitos e proposições presumidamente conhecidos e elaborar as proposições e questões instigadoras que conectam os pontos de partida aos pontos de chegada.

\section{Referências}

ALMEIDA, R. O. Ajofe e alcoometria: as escolas diante das mudanças socioculturais ligadas à produção de cachaça artesanal na microrregião de Abaíra, Bahia, Brasil. Ciência \& Educação, Bauru, v. 18, n. 1, p. 187-214, 2012.

AUSUBEL, D. P.; NOVAK, J. D.; HANESIAN, H. Psicologia educacional. 2. ed. Rio de Janeiro: Interamericana, 1978.

BLOOM, B. S. The 2 sigma problem: the search for methods of group instruction as effective as one-to-one tutoring. Educational Researcher, Abingdon, p. 4-16, 1984.

BLOOM, B. S.; KRATHWOHL, D. R. Taxonomy of educational objectives : the classification of educational goals. Handbook I, cognitive domain. New York: Longman, 1956.

CANTO, A. B. et al. Evasão e retenção em cursos de engenharia. In: SCHWERTL, S. L. et al. (Ed.). Desafios da educação em engenharia: vocação, formação, exercício profissional, experiências metodológicas e proposições. Blumenau: Edifurb, 2011. v. 1.

Tecnologia, descoberta e recepção: um estudo de caso. In: CONGRESSO

BRASILEIRO DE EDUCAÇÃO EM ENGENHARIA, 41., 2013, Gramado. Anais...

Gramado: 2013.

PBTA - Projeto baseado em trajetórias de aprendizagem. RENOTE: revista novas tecnologias na educação, v. 12, n. 1, 2014.

CANTO, A. B.; DE LIMA, J. V.; TAROUCO, L. Projeto e uso de objetos de aprendizagem: explorando as dimensões afetiva e cognitiva. Revista Iberoamericana de Educación en Tecnología y Tecnología en Educación, La Plata, v. 14, p. 7-17, 2014.

KIRSCH-PINHEIRO, M.; DE LIMA, J. V.; BORGES, M. R. S. A framework for awareness support in groupware systems. Computers in Industry, Amsterdam, v. 52, n. 1, p. 47-57, 2003.

KRATHWOHL, D. R. A revision of Bloom's taxonomy: an overview. Theory into Practice, Philadelphia, v. 41, n. 4, p. 212-218, 2002.

LABURÚ, C. E.; BARROS, M. A.; SILVA, O. H. M. Multimodos e múltiplas representações, aprendizagem significativa e subjetividade: três referenciais conciliáveis da educação científica.

Ciência \& Educação, Bauru, v. 17, n. 2, p. 469-487, 2011.

LODER, L. L. Engenheiro em formação: o sujeito da aprendizagem e a construção do conhecimento em engenharia elétrica. [Porto Alegre]: Universidade Federal do Rio Grande do Sul, 2009. 
MAYER, R. E. Applying the science of learning: evidence-based principles for the design of multimedia instruction. The American Psychologist, Washington, v. 63, n. 8, p. 760-769, 2008. MAYER, R. E.; MORENO, R. Nine ways to reduce cognitive load in multimedia learning. Educational Psychologist, Philadelphia, v. 38, n. 1, p. 43-52, mar. 2003.

MEC/SESU/DIFES. Reuni 2008 - relatório de primeiro ano, 2009. Disponível em: <http:// portal.mec.gov.br/index.php?option=com_docman\&task=doc_download\&gid=2069>. Acesso em: 30 maio. 2015.

MORENO, R.; MAYER, R. Interactive multimodal learning environments. Educational Psychology Review, New York, v. 19, n. 3, p. 309-326, 22 jun. 2007.

PRINCE, M. J.; FELDER, R. M. Inductive teaching and learning methods: definitions, comparisons, and research bases. Journal of Engineering Education, Hoboken, v. 95, n. 2, p. $123-138,2006$.

SWELLER, J. Cognitive load during problem solving: effects on learning. Cognitive Science, Cognitive Science, v. 12, n. 2, p. 257-285, jun. 1988.

TAVARES, R. Construindo mapas conceituais. Ciências \& Cognição, João Pessoa, v. 12, p. 72-85, 2007.

VAN MERRIËNBOER, J. J. G.; SWELLER, J. Cognitive load theory in health professional education: design principles and strategies. Medical Education, Chichester, v. 44, n. 1, p. 85-93, jan. 2010. 